\title{
Localización óptima de zonas potenciales para cosecha de agua lluvia mediante criterio booleano en la zona sur de Honduras, año 2017
}

José Cáceres Coello

\section{Resumen}

En Honduras 137 de los 298 municipios son considerados vulnerables a la sequía según el Plan de Acción Nacional de Lucha contra la Desertificación y la Sequía (PANLCD) que es el instrumento técnico y estratégico elaborado en el año 2005. La captación de agua de lluvia, también denominada "cosecha de agua", puede ser una de las soluciones para enfrentar el reto que plantea la baja disponibilidad del líquido en diversas regiones del país. Seis variables en criterio booleano fueron usados en esta investigación para seleccionar los sitios óptimos para la construcción de sistemas de cosecha y conservación de agua dentro del área de estudio. Se seleccionaron cinco municipios como área de estudio, todos en el departamento de Valle, y se encontró que las áreas óptimas para el establecimiento de cosechas de agua en la zona de estudio cubren un área de 3,529 Ha, lo cual representa un $5 \%$ del total del área.

Palabras Clave: Ponderación Booleana; Sistemas de Información Geográfica; Cosechas de Agua; Corredor Seco.

\section{Abstract}

In Honduras, 137 of the 298 municipalities are considered vulnerable to drought under the National Action Plan to Combat Desertification and Drought (PANLCD), which is the technical and strategic instrument developed in 2005. Rainwater harvesting, also known as "water harvesting", may be one of the solutions to meet the challenge posed by the low availability of liquid in various regions of the country. Six variables in Boolean criteria were used in this research to select optimal sites for the construction of water harvesting and conservation systems within the study area. Five municipalities were selected as the study area, all in the department of Valle, and it was found that the optimal areas for establishing water harvests in the study area cover an area of $3,529 \mathrm{Ha}$, which represents $5 \%$ of the total area.

Keywords: Boolean weighting; Geographic information systems; Water Harvesting; Dry Corridor. 
José Cáceres Coello Facultad de Ciencias Espaciales. Departamento de Ciencia y Tecnologías de la Información Geográfica. Universidad Nacional Autónoma de Honduras. Edificio K2, Ciudad Universitaria, Tequcigalpa M.D.C. Teléfono: 2216-3034. email: jcaceres@unah.edu.hn

Fecha de Recepción: 20 de agosto del 2018 / Fecha de Aprobación: 02 de noviembre del 2018 


\section{INTRODUCCIÓN}

En Honduras 137 de los 298 municipios son considerados vulnerables a la sequía según el Plan de Acción Nacional de Lucha contra la Desertificación y la Sequía (PANLCD) que es el instrumento técnico y estratégico elaborado en el año 2005 (FAO (2014)).

De acuerdo al Informe de Evaluación de Impacto de la Sequía (FAO (2014)), en relación a las pérdidas de granos básicos en el ciclo de primera del 2014, se define que las familias en condiciones de extrema pobreza del Corredor Seco, son las más afectadas, familias que producen para la subsistencia y sin apoyo adicionales para la producción.

Actualmente el Gobierno de Honduras, a través del Ministerio de Agricultura y Ganadería, tiene planeado desarrollar una fuerte inversión en proyectos de cosecha de agua para mitigar los efectos de la sequía prolongada, sin embargo, la selección de estos sitios no responde a variables de localización espacial, ni se estudia la idoneidad de los sitios seleccionados, por lo cual el desarrollo de este proyecto permitirá la optimización de la inversión pública en este tipo de proyectos, mediante de la identificación y selección de sitios que provean el mayor potencial de captación de agua lluvia (Coello (2004)).

En el año 2015 los problemas derivados por la Sequía en el Corredor Seco Hondureño se han agravado, según un informe de la (FAO (2014)), Honduras está en la lista de cuatro países centroamericanos que urgen ayuda internacional para paliar los efectos derivados por la prolongada canícula. Este es el segundo año consecutivo en que la cosecha de cereales (maíz) de la temporada principal en la región se ha visto afectada negativamente por la grave sequía relacionada con El Niño.

El fenómeno de la sequía es, entre las incertidumbres geográficas, la que ocasiona mayores pérdidas de producción en las regiones sin riego y, en muchas ocasiones, también en las que cuentan con él. La desertificación consiste en una degradación persistente de los ecosistemas de las tierras secas producida por las variaciones climáticas y la actividad del hombre. Las Cosechas de Agua son las captaciones de las aguas pluviales en las quebradas de invierno. Cada sistema implica estudios, diseño y ejecución de las obras hidráulicas y embalses para la captación y almacenamiento de aguas pluviales y líneas de conducción, así como el desarrollo y adopción de sistemas de riego de tipo complementario a nivel de la parcela de cada productor.

La captación de agua de lluvia puede ser una de las soluciones para enfrentar el reto que plantea la baja disponibilidad del líquido en diversas regiones del país donde la escasez de agua para consumo es producto de la falta de infraestructura para almacenamiento y de distribución del agua, la contaminación del agua superficial y subterránea y la ingobernabilidad en la gestión de este sector. Al captar y usar el agua de lluvia, con una tecnológica adecuada se puede contar con alternativas para el abastecimiento de agua a un bajo costo. La captación de agua de lluvia, también denominada "cosecha de agua" consiste en colectar el agua de las precipitaciones pluviales en una superficie para su almacenamiento y posterior uso (Coello (2004)).

Para identificar los sitios óptimos para el establecimiento de cosechas de agua en la zona sur del país se abordarán los siguientes objetivos específicos:

1. Identificar el grado de amenaza a sequía en la zona sur de Honduras. 
2. Caracterizar las zonas óptimas para la implementación de cosechas de agua en base a consideraciones binarias.

\section{ANTECEDENTES}

El agua es uno de los recursos naturales más importantes de las regiones áridas del mundo (Allison, 1998) y hay zonas del mundo que tienen una grave escasez de agua. La escasez de agua, tradicionalmente, ha restringido el desarrollo donde el mismo se basa en suministros suficientes, fiables y duraderos en términos de cantidad y calidad (Dottridge y Gibbs (1998))

El Corredor Seco de Honduras se caracteriza por precipitaciones irregulares, además, los períodos de canícula son más extensos, y se intensifican cuando está presente el fenómeno de EI Niño, porque se reducen las lluvias entre un $30-40 \%$. En el $79 \%$ de los 1,800 casos, dura 2 meses o menos, y solo en los eventos más críticos, la anomalía es aumentada por un déficit de precipitación total anual o se prolonga a todo el período de la postrera (agosto a octubre) (FAO (2014)).

Durante las épocas de sequía la producción de estos cultivos se ve fuertemente afectadas en calidad y cantidad, el fenómeno de El Niño Oscilación del Sur, ha afectado intensamente con eventos de sequía prolongados, en especial en la región del Pacífico, en la cual, y en gran medida cultiva granos básicos, producción de camarón, ganadería y aves, esta última en menor cantidad. Se puede decir que el fenómeno de El Niño es el mayor causante de pérdidas en el sector agrícola en la región Sur, y Sur-Occidente de Honduras lo que representa un impacto a la economía hondureña.

Sin embargo, vale la pena mencionar que estas pérdidas a pesar de tener como detonante a la sequía son asociadas al mal manejo de los recursos naturales. Así mismo otras amenazas como inundaciones y deslizamientos también son potenciales contribuyentes a estas pérdidas. En el último siglo el país ha sufrido la ocurrencia de 27 huracanes, tormentas tropicales y variados desastres naturales con un aproximado de 4.7 millones de personas afectadas.

Los departamentos de Gracias a Dios, Colón, Atlántida, Cortés y Yoro están mayormente expuestos a desastres por inundaciones. Por otro lado, los departamentos como Valle, Choluteca, La Paz, Comayagua son proclives a sufrir inundaciones pero también sequías, especialmente afectando a los pobres (FAO (2014)).

La búsqueda de nuevos recursos de agua tiene una alta prioridad en el país debido a la situación actual de los recursos hídricos disponibles. Entre estos recursos es la captación de agua por las presas y estanques. Captación de agua se aplica en las regiones áridas y semiáridas donde las precipitaciones son o no suficientes para mantener un buen crecimiento de los cultivos y pastos o cuando, debido a la naturaleza errática de precipitación, el riesgo de fracaso de la cosecha es muy alta (Prinz y Singh (2000)).

Antes de la construcción de nuevos esquemas de recolección de agua, los sitios óptimos para estos esquemas deben ser cuidadosamente seleccionados en base a los parámetros físicos y socio-económicos que caracterizan la zona específica. La capacidad de los SIG para manipular los datos geográficamente referenciados da a los tomadores de decisiones en una excelente herramienta para 
Localización óptima de zonas potenciales para cosecha de agua lluvia mediante criterio booleano en la zona sur de

elegir los mejores sitios para los esquemas de recolección de agua.

Los aspectos físicos e hidrológicos de la captación de agua se han explorado a través del uso de sistemas de información geográfica (SIG) y el modelado hidrológico en varios estudios. (Srivastava (1996)) mencionó que, para los pequeños embalses de riego, la selección del sitio se basa en consideraciones hidrológicas, topográficas y socioeconómicas. (Vorhauer y Hamlett (1996))utilizaron un enfoque de SIG para localización de estanques de riego para uso agrícola. Los criterios de selección de sitios utilizados en sus investigaciones incorporan suelo e idoneidad de pendiente, la cobertura del suelo actual y el uso del suelo en la zona. (Nisar Ahamed y Murthy (2008)) desarrollaron un algoritmo basado en SIG para determinar la ubicación de los puntos de venta para las pequeñas cuencas para determinar los posibles emplazamientos para embalses. Los investigadores utilizaron un mapa de drenaje y una rejilla-DEM como entrada.(Baban y Wan Yusof (2003))desarrolló, aplicó y evaluó un criterio de selección de sitios que incluyeron la hidrología y la hidráulica, la topografía, la geología, la economía, y las implicaciones ambientales para seleccionar los sitios de depósito adecuados a gran escala en ambientes tropicales mediante teledetección y SIG como herramienta de ayuda a los tomadores de decisiones. (Forzieri, Gardenti, Caparrini, y Castelli (2008)) utilizaron los SIG como herramienta para la pre-selección de sitios adecuados para la superficie $\mathrm{y}$ pequeñas presas subterráneas en zonas áridas de la región de Kidal, Malí. (Chang y Breeden (2008)) combinaron SIG y evaluación multicriterio difusa para el emplazamiento de vertedero en una región urbana de rápido crecimiento en el Valle del Río Grande Bajo, Texas, EE.UU. (KaIlali, Anane, Jellali, y Tarhouni (2007)) utilizaron un análisis multicriterio basado en SIG para los posibles sitios de recarqa de acuíferos de aquas residuales en la parte nororiental de Túnez. (Gemitzi, Tsihrintzis, Christou, y Petalas (2007)) utilizaron los SIG en emplazamiento instalaciones de estanques de estabilización para el tratamiento de aguas residuales domésticas en Grecia. El uso de los SIG para la ubicación de esquema de recolección de aqua se ha explorado también por otros investigadores (Ramalingam y Santhakumar (2000)).

Esos investigadores utilizaron los SIG para seleccionar sitios adecuados para esquemas de recolección de agua (estanques y presas) en diferentes partes del mundo. Se han utilizado diferentes capas de SIG, incluyendo el uso del suelo, las carreteras, la topografía y el suelo.

\section{III ÁREA DE ESTUDIO}

El área de estudio abarca los municipios de Caridad, Aramecina, Goascorán, Langue y Alianza, del Departamento de Valle (Sur de Honduras), específicamente en la parte baja de la cuenca del Río Goascorán, y cubre un área total de 68,533 hectáreas, lo que representa aproximadamente un $42 \%$ de la extensión total del Departamento (Figura 1), y se encuentra habitada por 54,699 habitantes distribuidos en 343 caseríos (2013). 


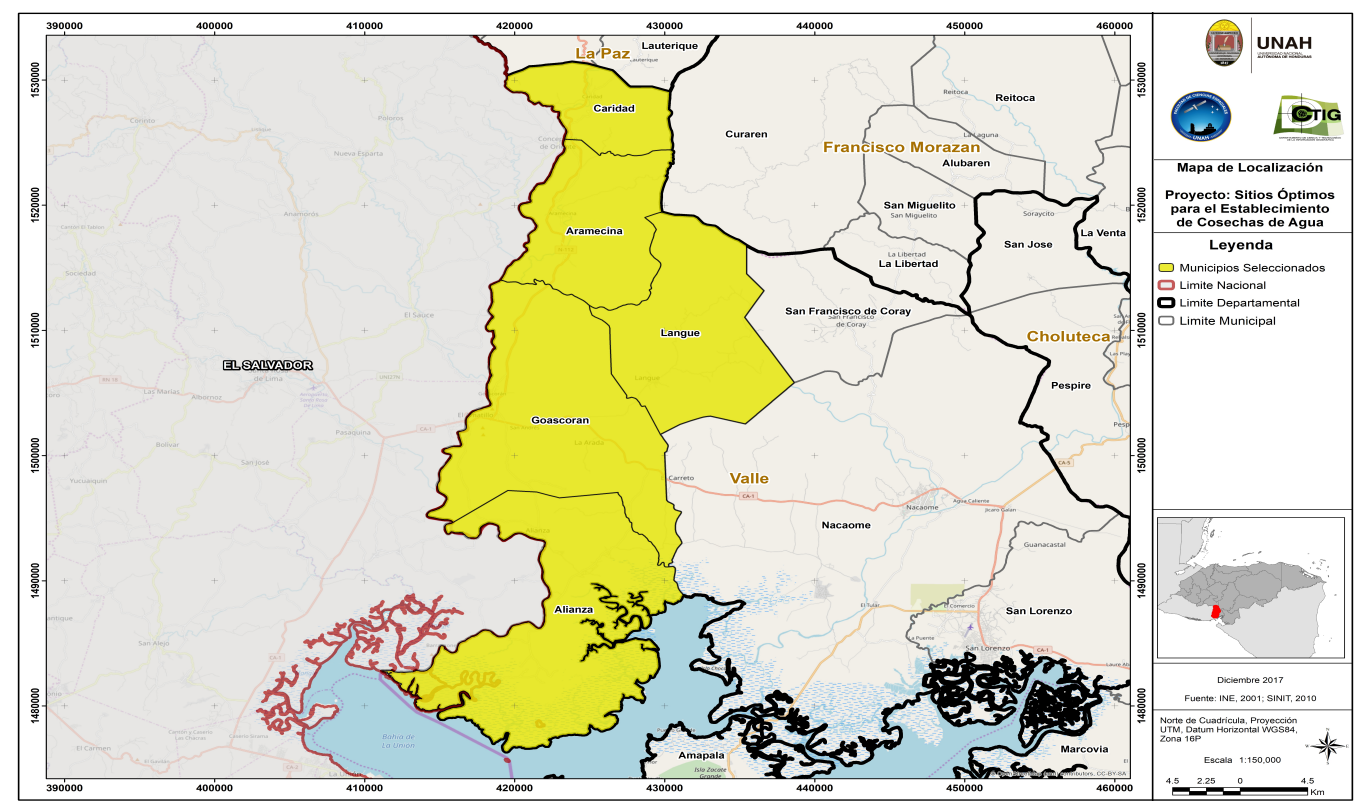

Figura 1: Área de Estudio. Elaboración propia

El tipo de drenaje es dendrítico con mayor densidad en la parte alta y media que definen ocho subcuencas con ríos de gran importancia que conforman la red hídrica de la misma. La cobertura natural vegetal se caracteriza por encontrar rodales de pino en asociación con especies menores como nance, roble y encino en la zona alta de la cuenca, la zona media se caracteriza por el desarrollo de actividades productivas, como ganadería, agricultura y la pesca artesanal. En la zona baja encontramos zonas con pendientes menores al $10 \%$ con vegetación característica de bosques xerofíticos caducifolios en la generalidad de las especies.

En la zona baja de la cuenca, la red hídrica está conformada por los ríos Las Pilas, Agua Caliente, y Santa Rosa, que son tributarios del Río Pasaquina, formado por la unión del Río Santa Rosa y Agua Salada y a la vez, el río Pasaquina es tributario del Río Goascorán. El río Guajiniquil hasta su confluencia con el Río Goascorán sirve de Línea Divisoria entre los dos países, de manera que la subcuenca del Río Guajiniquil es la única binacional, pues a ella drenan aguas de los dos territorios. Por lo general, a estos ríos principales se unen pequeños riachuelos y quebradas de menor importancia, los cuales constituyen el sistema de drenaje natural tipo dendrítico, cuyas longitudes en sus cauces varían de 12 a $51 \mathrm{Km}$.

En la parte baja de la cuenca, las especies vegetales que se encuentran están relacionadas con los sistemas acuáticos, formados por manglares y humedales y vegetación nativa dispersa entre los que se encuentra el quebracho, madre cacao, guanacaste, laurel y almendro de río. Dentro de los manglares tenemos el más común como el Ryizophora mangle y Ryizophora racemosa comúnmente llamado como mangle colorado. Tambièn se pueden observar especies introducidas a orillas de la carretera, siendo ellas el Terebinto, Teca, eucalipto, paraíso, leucaena, mamón, almendro de playa, flor de fuego y cerezo de Belice o carao.

En la actualidad la fauna se encuentra muy escasa, según la percepción de los habitantes es debido a la caza y a la deforestación por quemas agrícolas, sin em- 
Localización óptima de zonas potenciales para cosecha de agua lluvia mediante criterio booleano en la zona sur de

bargo, los avistamientos de las pocas especies existentes ocurren principalmente durante la noche debido a los hábitos nocturnos de las mismas. En el contexto territorial de la zona de estudio ha sido identificada un área natural protegida, que por la importancia de los recursos naturales que posee, ha sido incluida en los planes de conservación del Sistema Nacional de Áreas Protegidas. Es necesario recalcar la importancia de lograr un desarrollo sostenible en la cuenca y específicamente en la parte baja de la misma, para lo cual se vuelve fundamental preservar los ambientes propicios para asegurar la disponibilidad de recursos naturales en el área.

El área protegida encontrada dentro de nuestra zona de estudio es la Bahía de Chismuyo, la cual abarca parte del municipio de Alianza, y la misma comprende un área de aproximadamente $282 \mathrm{Km} 2$, en una zona de vida catalogada como bosque húmedo montano bajo. Es un área conformada de rodales de manglares que son el hábitat de reptiles y mamíferos, crustáceos, aves, moluscos y peces. Actualmente se da una expansión y establecimiento de fincas camaroneras, deforestación por uso de madera y leña y expansión ganadera, lo cual genera grandes presiones al área protegida (Moreno Segura, Mondragón Rivera, Cáceres Coello, y Carias Arias (2016)).

\section{MEDOTOLOGÍA}

El alcance del estudio es la delimitación de zonas óptimas para el desarrollo de proyectos de cosecha de agua en la zona sur de Honduras.

El enfoque del estudio es estrictamente cuantitativo, ya que se pretende cuantificar la cantidad de área adecuada para el desarrollo de proyectos de cosecha de agua. Basado en un tipo de estudio correlacional, mediante la construcción de un modelo espacial multicriterio a partir de las variables seleccionas para determinar la idoneidad de una zona para captar agua lluvia.

La población del estudio lo conforma el territorio de los municipios, que serán priorizados, pertenecientes al departamento de Valle.

Se presenta a continuación un esquema metodológico que resume las fases del desarrollo del proyecto de investigación

\section{Delimitación concreta y clara de la zona de estudio.}

Se procederá a priorizar los municipios del departamento de Valle más afectados por los eventos de desertificación y sequía y se procederá posteriormente a delimitar el área de estudio.

2. Formulación de los criterios de localización a ser tenidos en cuenta en el proceso de análisis y resolución del problema. Para ello se realizará una revisión de la literatura sobre la cuestión.

En base a la revisión bibliográfica se procederá a definir las variables a utilizar para la selección de sitios óptimos para el desarrollo de proyectos de cosecha de agua, así como las posibles fuentes de generación de esa información.

3. Creación de la base de datos geográfica a utilizar en el análisis posterior.

Se procederá a recopilar la información necesaria y a espacializar las variables dentro de una base de datos geográfica que permita el análisis espacial de la misma. 
4. Análisis descriptivo de las variables territoriales planteadas para conocer bien la situación inicial. Diseño y realización de un conjunto cartográfico de todas las variables.

Se procederá a realizar un análisis descriptivo de la información geográfica recopilada y se procederá a generar la representación cartográfica necesaria, por variable, del área de estudio

5. Definición de un procedimiento de resolución del problema, basado en la revisión de la literatura, procedimiento que permitirá, mediante técnicas SIG y de evaluación multicriterio, definir las zonas más adecuadas para la localización de las instalaciones hidráulicas necesarias.

Se procederá a definir un modelo espacial que permita definir la conjugación de diferentes variables para identificar zonas adecuadas para la localización de las instalaciones hidráulicas.

6. Identificación y análisis detallado de las características de las zonas seleccionadas como más adecuadas. Elaboración de un conjunto cartográfico de los factores de localización y de las diversas soluciones encontradas.

Basado en criterios mínimos de selección se procederá a aglomerar las zonas óptimas identificadas, basado en tamaños mínimos requeridos para satisfacer necesidades en base a población, para identificar, seleccionar y cartografiar las zonas óptimas.

\section{RESULTADOS Y DISCUSIÓN}

El área de estudio seleccionada se basó en previos estudios realizados en la zona del corredor seco, específicamente en cinco municipios de la cuenca baja del Río Goascorán, pertenecientes al departamento de Valle (Figura 1). Estos municipios fueron clasificados como sequía moderada según el índice de sequía de Palmer. El área de estudio abarca los municipios de Caridad, Aramecina, Langue, Goascorán y Alianza, y cubre un área total de 68,533 hectáreas y se encuentra habitada por 54,699 habitantes distribuidos en 343 caseríos (2013).

La cobertura forestal (Figura 2) en el área de estudio se compone principalmente de bosque latifoliado seco (34\%), pastos y/o cultivos (30\%), vegetación secundaria seca (18\%), entre otros. Las precipitaciones varían de 1,780 a 2,060 mm anuales (Figura 3), encontrándose las mayores precipitaciones en la parte norte del área de estudio y en el sur en la zona costera. La elevación de la zona de estudio varía de cero en la zona costera localizada al sur del área de estudio hasta los 1,260 m.s.n.m. en la parte norte (Figura 4) La dirección del flujo de agua superficial es hacia el sur, siguiendo la pendiente natural del terreno (Figura 5). 


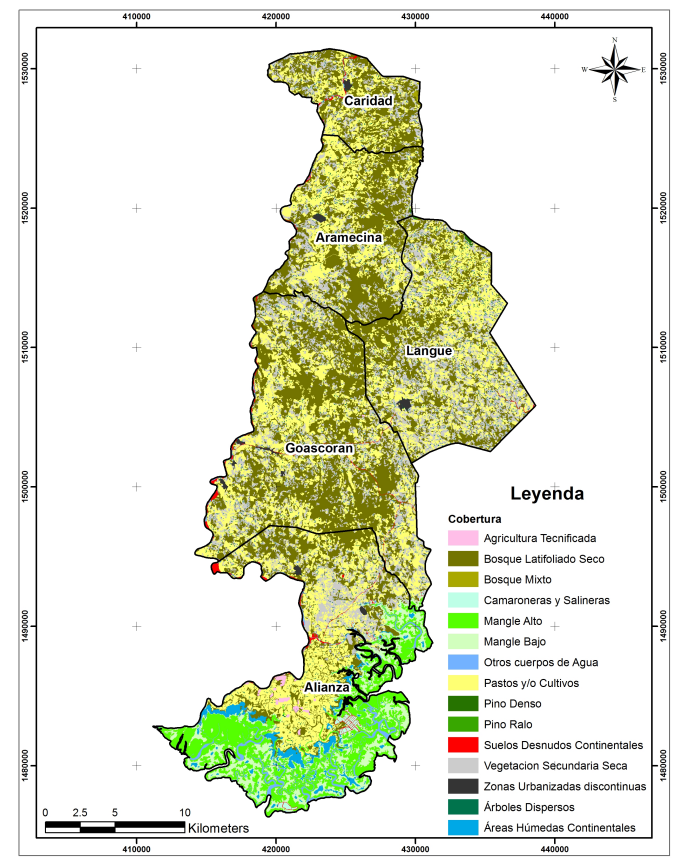

(a) Figura 2: Cobertura Forestal. Elaboración propia

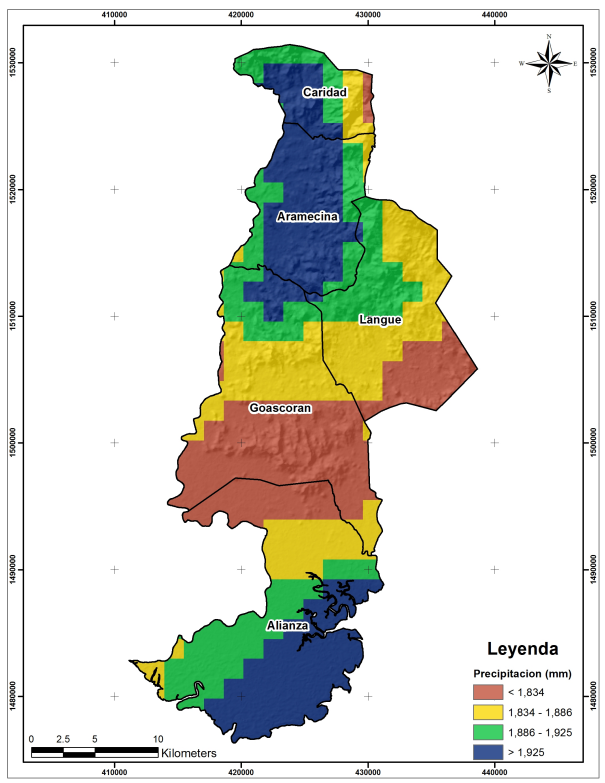

(b) Figura 3: Precipitaciones. Elaboración propia

Seis criterios en ponderación binaria fueron usados en esta investigación para seleccionar los sitios óptimos para la construcción de sistemas de cosecha de agua dentro del área de estudio. Estos criterios incluyen, precipitación anual, pendiente (\%), distancia a cuerpos de aqua, cobertura del suelo, distancias a centros urbanos y distancia a red vial, (Shatnawi (2016)) tal y como se muestra en la Tabla 1. 


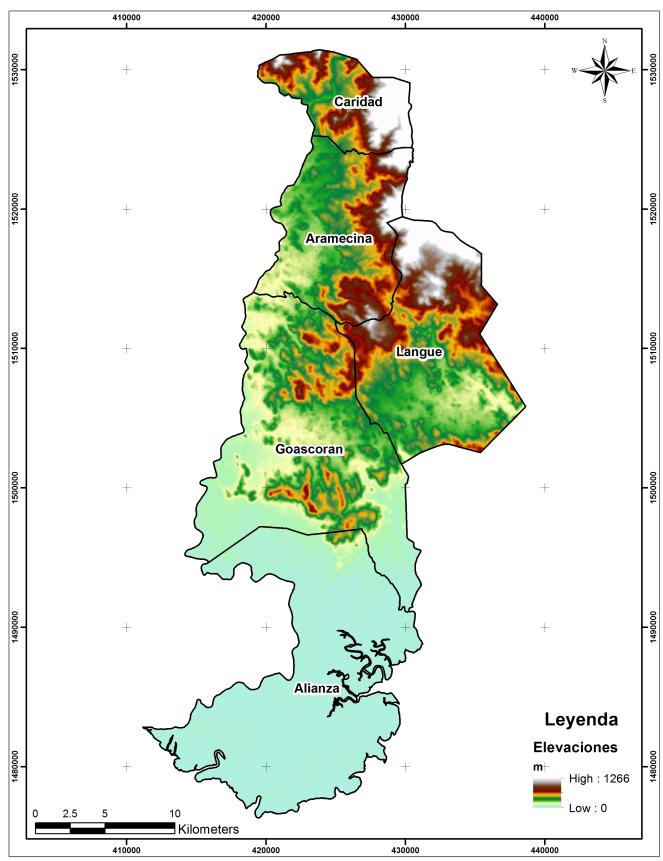

(a) Figura 4: Elevaciones. Elaboración propia

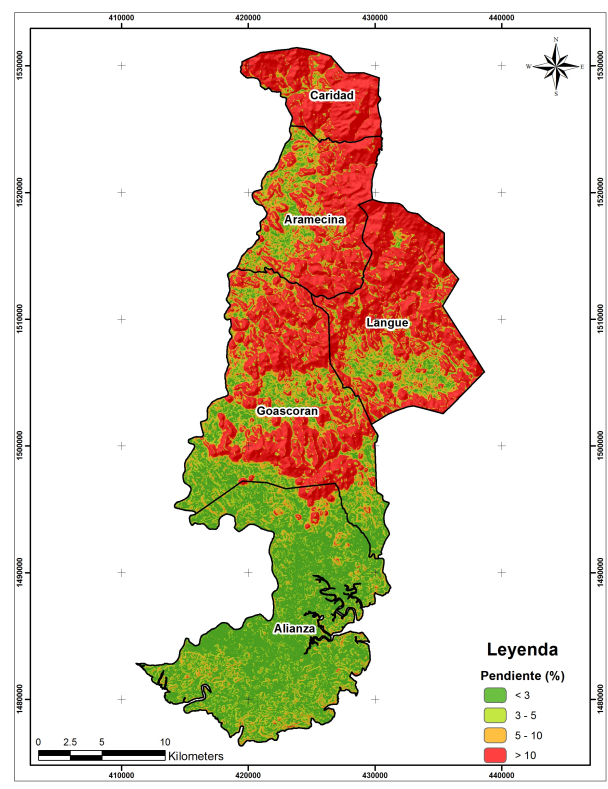

(b) Figura 5: Pendientes. Elaboración propia

Tabla 1: Pesos, Rangos y Ponderación de los seis criterios utilizados. Elaboración propia

\begin{tabular}{|l|c|c|}
\hline \multicolumn{1}{|c|}{ Parámetros } & Rangos & Ponderación \\
\hline $\begin{array}{l}\text { Precipitación } \\
\text { anual (mm) }\end{array}$ & $<1,886$ & 0 \\
& $>1,886$ & 1 \\
\hline Pendiente (\%) & $>5$ & 0 \\
& $<5$ & 1 \\
\hline $\begin{array}{l}\text { Cobertura del } \\
\text { suelo }\end{array}$ & $\begin{array}{c}\text { Bosque latifoliado seco, bosque mixto, pino denso, } \\
\text { de agua, camaroneras y salineras, Agricultura } \\
\text { tecnificada, zonas urbanizadas discontinuas }\end{array}$ & 0 \\
& $\begin{array}{c}\text { Pastos y/o cultivos, árboles dispersos, Vegetación } \\
\text { secundaria seca, suelos desnudos, áreas húmedas } \\
\end{array}$ & 1 \\
\hline $\begin{array}{l}\text { Distancia a } \\
\text { cauces (m) }\end{array}$ & $<1000$ & 1 \\
\hline $\begin{array}{l}\text { Distancia a } \\
\text { centros poblados } \\
\text { (m) }\end{array}$ & $>1,000$ & 0 \\
\hline $\begin{array}{l}\text { Distancia a red } \\
\text { vial }(\mathrm{m})\end{array}$ & $<1500$ & 1 \\
\hline
\end{tabular}




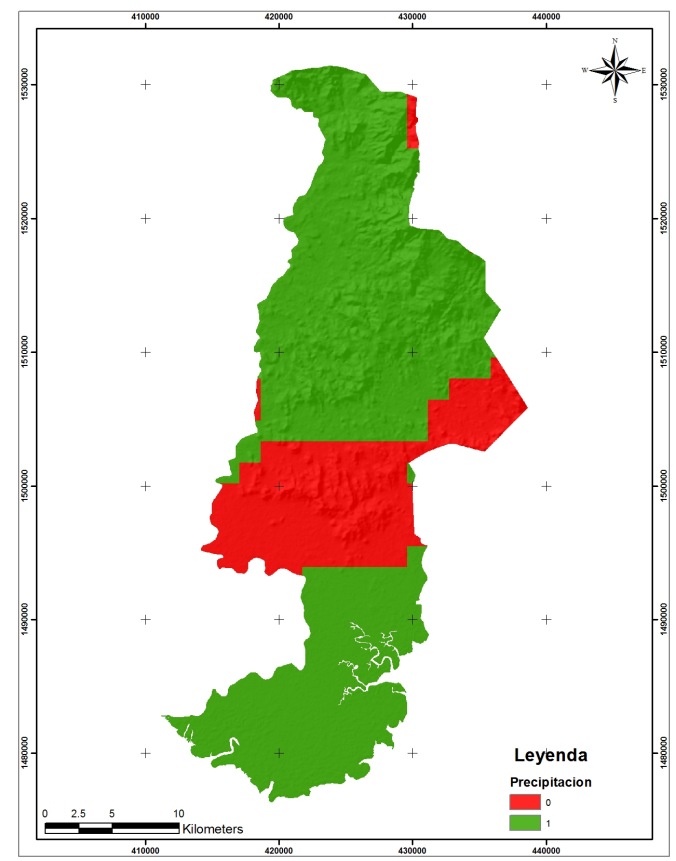

(a) Figura 6: Precipitación Anual. Elaboración propia

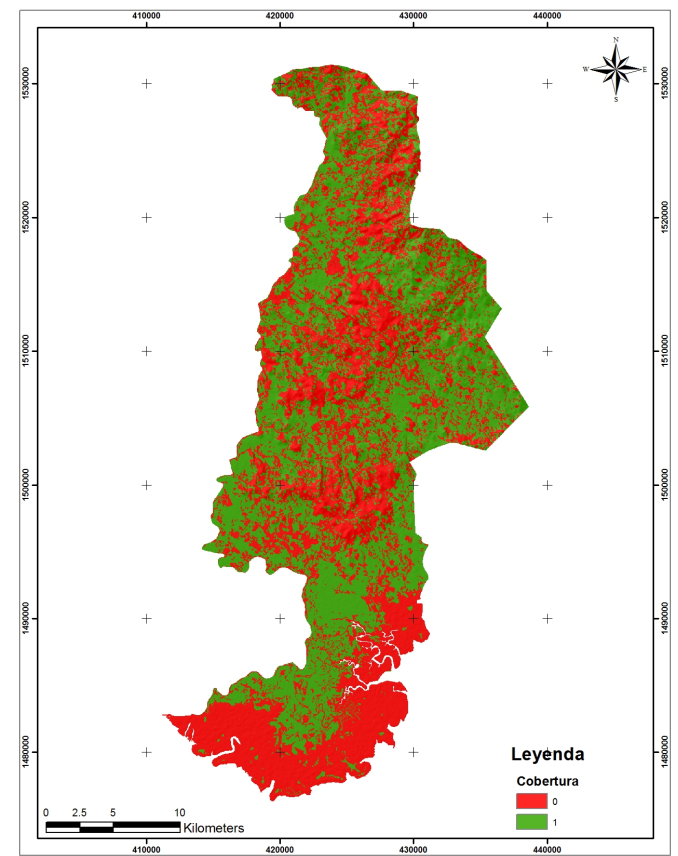

(a) Figura 8: Cobertura del Suelo. Elaboración propia

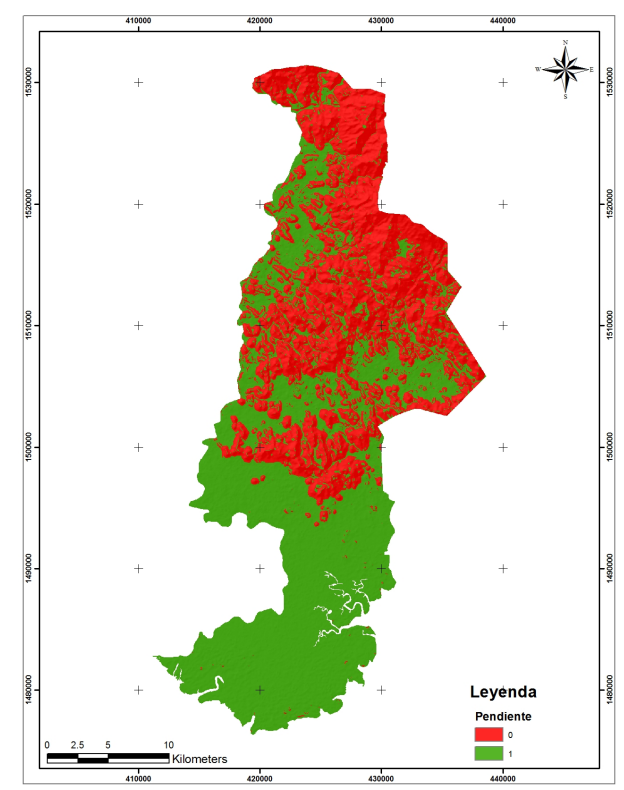

(b) Figura 7: Pendientes. Elaboración propia

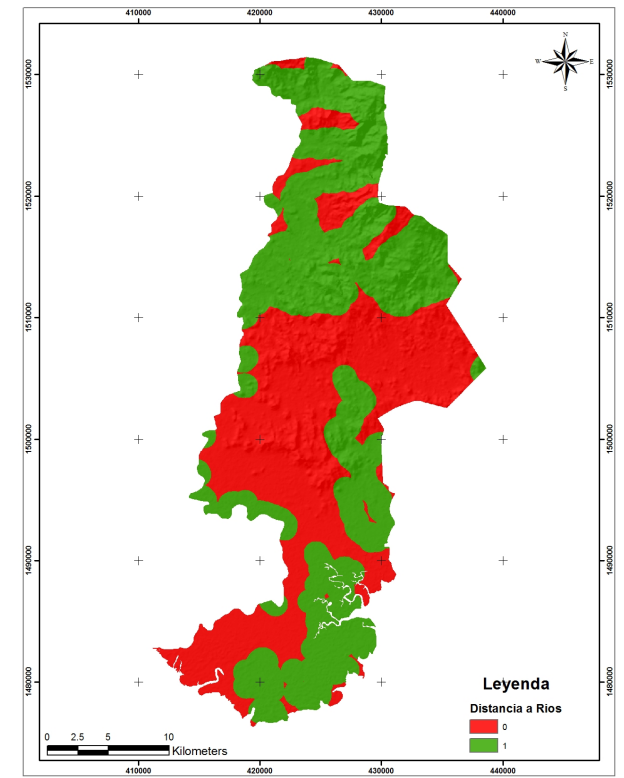

(b) Figura 9: Distancia a Cauces. Elaboración propia 


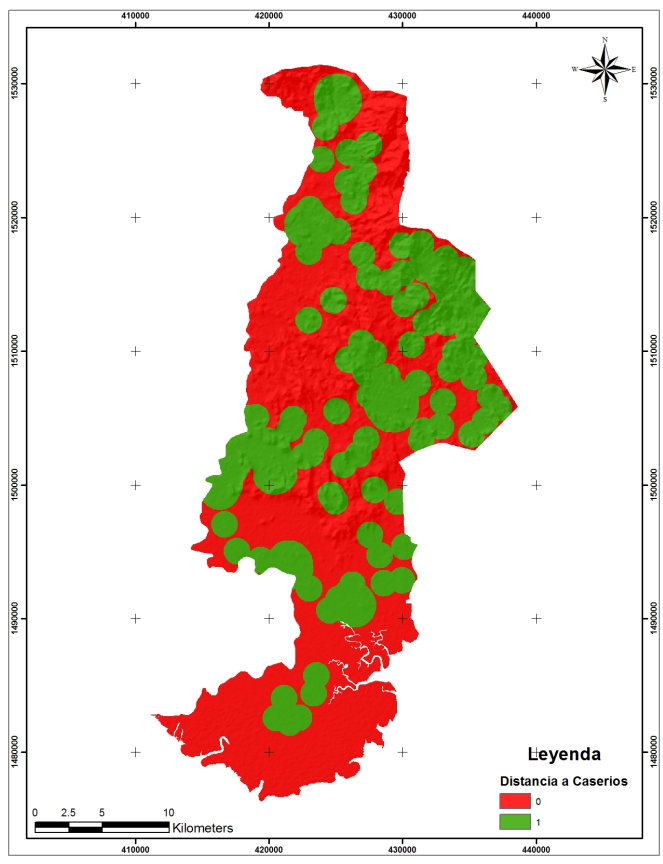

(a) Figura 10: Distancia a Centros Poblados. Elaboración propia

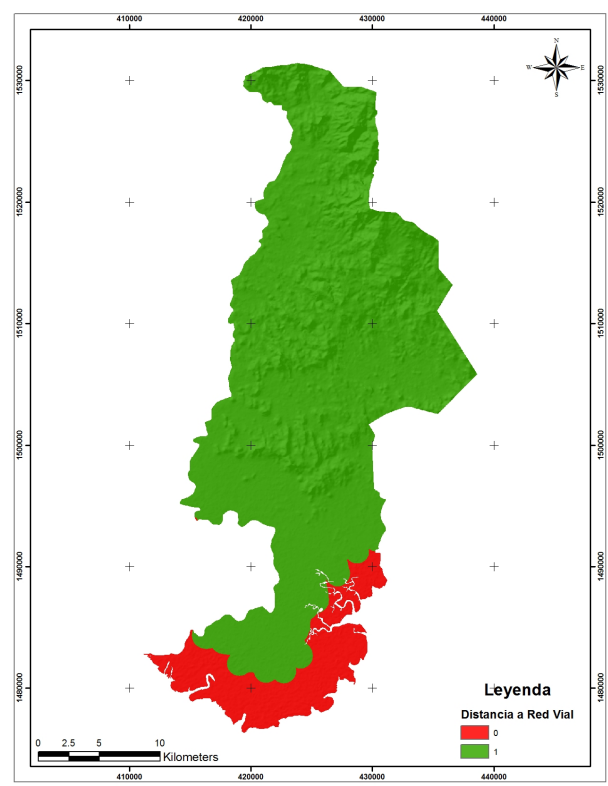

(b) Figura 11: Distancia a Red Vial. Elaboración propia

Los criterios utilizados fueron implementados utilizando programas especializados en el manejo de información geográfica, mediante el cual se utilizaron procesos de reclasificación para definir la ponderación pertinente para cada categoría. En las Figura 6 a Figura 11, se muestran los resultados obtenidos al realizar los procesos de reclasificación.

En esta investigación, se encontró que el valor mínimo calculado por el método de ponderación booleana fue de 0 , mientras que el máximo fue de 1 . En base a los valores obtenidos se definieron dos categorías, como se muestra en la Tabla 2. 
Tabla 2: Clasificación del Nivel de Adecuación y el Resultado Final del método WLC. Fuente: Elaboración propia

\begin{tabular}{|c|c|c|c|}
\hline Clase & $\mathbf{0}$ & $\mathbf{1}$ & Total \\
\hline Nivel de Adecuación & No adecuado & Adecuado & \\
\hline Área (ha) & $64,998.08$ & $3,529.28$ & $68,527.36$ \\
\hline \% del Total del Área & 94.85 & 5.15 & 100 \\
\hline
\end{tabular}

Se encontró que las áreas óptimas para el establecimiento de cosechas de agua en la zona de estudio cubren un área de 3,529.28 Ha (Figura 12), lo cual representa un $5.1 \%$ del total del área (Al-Adamat, 2008), mientras que las áreas restantes se clasifican como No Adecuado (94.85\%).

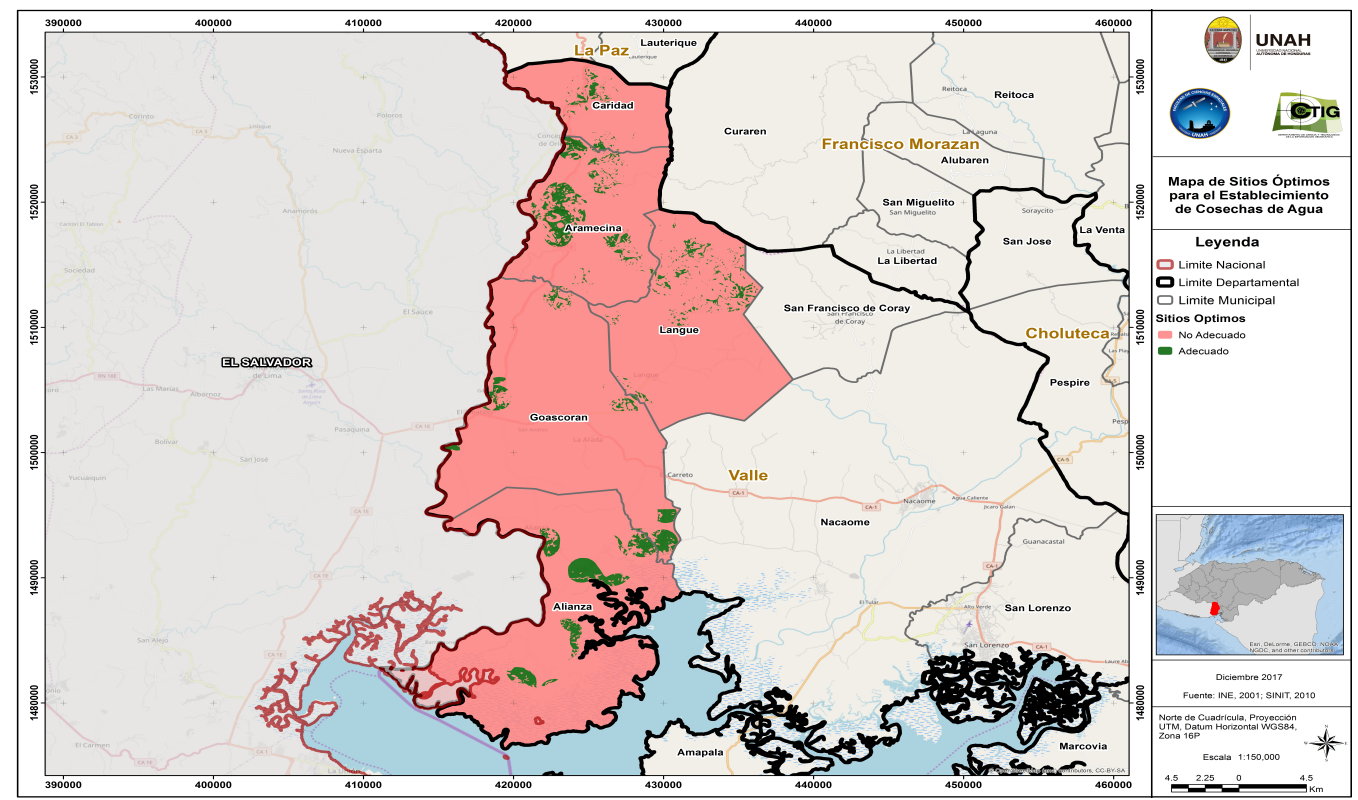

Figura 7: Mapa de Localización de Sitios Óptimos. Elaboración propia

\section{CONCLUSIONES}

El corredor seco de Honduras, específicamente los municipios seleccionados pertenecientes al Departamento de Valle, es catalogado como una zona muy seca y con escasez de agua, sin embargo, tal y como se ha encontrado en este estudio, las precipitaciones de la zona son considerables, por lo cual se evidencia 
una falta de infraestructura para retener y aprovechar sosteniblemente el recurso agua de la zona. Los proyectos de cosecha de agua han sido utilizados en zonas donde no se posee infraestructura de gran envergadura, como alternativas viables para aprovechar las precipitaciones de la zona.

En esta investigación se han utilizados varias técnicas de análisis espacial para seleccionar los sitios óptimos para el establecimiento de cosechas de agua en los municipios de Caridad, Aramecina, Goascorán, Langue y Alianza.

El método de Combinación Lineal Ponderada (WLC), con enfoque binario, se aplicó sobre seis criterios espaciales resultando en la identificación de un $5 \%$ del total del área de estudio $(3,529.28 \mathrm{Ha})$ con un potencial alto para la construcción de cosechas de agua.

En conclusión, esta investigación demostró las poderosas capacidades de los SIG en el manejo de datos digitales para seleccionar los sitios óptimos para las cosechas de agua en la cuenca baja del Río Goascorán. El corredor seco, con sus recursos hídricos limitados, tiene una necesidad de implementar herramientas científicas que ayuden a los tomadores de decisiones, en diversos niveles gubernamentales, a combatir la escasez de agua, ahorrando dinero y tiempo para explorar áreas para la selección de sitios óptimos para cosechas de agua.

Por último, se recomienda que los organismos gubernamentales utilicen el SIG para seleccionar los sitios óptimos para los proyectos de cosecha de agua en todo el país. Asimismo, se recomienda que la metodología adoptada en esta investigación se desarrolle a través de la integración de los conocimientos indígenas que puedan conducir a una mejor selección del sitio. Además, se recomienda llevar a cabo trabajos de campo en los sitios seleccionados para una investigación más profunda para asegurarse de que los sitios seleccionados no estén en conflicto con otros usos de la tierra que no se muestran al investigador a través de los datos SIG disponibles.

\section{Referencias}

Baban, S., y Wan Yusof, K. (2003). Modelling optimum sites for locating reservoirs in tropical

Chang, G., N. B.and Parvathinathan, y Breeden, J. B. (2008). Combining gis with fuzzy multicriteria decision-making for landfill siting in a fast-growing urban region. research support, non-u.s. gov't. doi: 10.1016/j.jenvman.2007.01.011

Coello, J. C. (2004). Localización óptima de zonas potenciales para cosecha de agua lluvia en la zona sur de honduras, año 2016. doi: http://dx.doi.org/10.5377/ce.v10i1.5819

Dottridge, J., y Gibbs, B. (1998). Arid land resources and their management, jordan desert margin.

FAO. (2014). Aplan de acción nacional de lucha contra la desertificación (pan-Icd) 2014-2022. Honduras.

Forzieri, G., Gardenti, M., Caparrini, F., y Castelli, F. (2008). methodology for the pre-selection of suitable sites for surface and underground small dams in arid areas: $A$ case study in the region of kidal, mali. doi: 10.1016/j.pce.2007.04.014

Gemitzi, A., Tsihrintzis, V. A., Christou, O., y Petalas, C. (2007). Use of gis in siting stabilization pond facilities for domestic wastewater treatment. research support, non-u.s. gov't. doi: 10.1016/j.jenvman.2005.12.022

Kallali, H., Anane, M., Jellali, S., y Tarhouni, J. (2007). Gis-based multi-criteria analysis for potential wastewater aquifer recharge sites. doi: 10.1016/j.desal.2006.11.016

Moreno Segura, E., Mondragón Rivera, C., Cáceres Coello, J., y Carias Arias, A. (2016). dentificación de riesgos por desastres naturales en las cuencas hidrográficas matasnillo (panamá) y goascorán (honduras), y su relación con el cambio climático utilizando tecnologías de información geográfica. doi: http://dx.doi.org/10.5377/ce.v9i1.3126 
Nisar Ahamed, K., T. R.and Gopal Rao, y Murthy, J. S. R. (2008). Automatic extraction of tank outlets in a sub-watershed using digital elevation models. doi: 10.1016/S0378-3774(02) $00047-1$.

Prinz, D., y Singh, A. K. (2000). Water resources in arid regions and their sustainable management. annals of arid lands.

Ramalingam, M., y Santhakumar, A. R. (2000). Case study on artificial recharge using remote sensing and gis. Retrieved 11/26/2015. Descargado de http://geospatialworld.net/ Paper/Application/ArticleView . aspx?aid=1276

Shatnawi, G. (2016). Determine the best sites for water harvesting projects (dams \& hafirs) in northeastern badia using gis applications. Unpublished M.Sc. thesis, Al Al-Bayt University, Mafraq.

Srivastava, R. (1996). Methodology for optimizing design of integrated tank irrigation system. doi: 10.1061/(ASCE)0733-9496(1996)122:6(394)

Vorhauer, C., y Hamlett, J. (1996). Gis; a tool for siting small farm ponds. Cartagena . Bogotá. Colombia. 